\title{
GRam stain-guided Antibiotics ChoicE for Ventilator-Associated Pneumonia (GRACE-VAP) trial: rationale and study protocol for a randomised controlled trial
}

\author{
Jumpei Yoshimura ${ }^{1}$, Kazuma Yamakawa ${ }^{1 *}$ (D), Takahiro Kinoshita ${ }^{1}$, Yoshinori Ohta ${ }^{2}$ and Takeshi Morimoto ${ }^{3}$
}

\begin{abstract}
Background: Optimising the use of antibiotic agents is a pressing challenge to overcoming the rapid emergence and spread of multidrug-resistant pathogens in intensive care units (ICUs). Although Gram staining may possibly provide immediate information for predicting pathogenic bacteria, Gram stain-guided initial antibiotic treatment is not well established in the ICU setting. We planned the GRam stain-guided Antibiotics ChoicE for VentilatorAssociated Pneumonia (GRACE-VAP) trial to investigate whether Gram staining can safely restrict the use of broad-spectrum antibiotics in patients with ventilator-associated pneumonia (VAP), which is one of the most common hospital-acquired infections in ICUs.
\end{abstract}

Methods/design: The GRACE-VAP trial is a multicentre, randomised, open-label parallel-group trial to assess the non-inferiority of Gram stain-guided initial antibiotic treatment to guidelines-based initial antibiotic treatment for the primary endpoint of clinical response rate in patients with VAP. Secondary endpoints include the coverage rates of initial antibiotic therapies, the selected rates of anti-pseudomonal agents and anti-methicillin-resistant Staphylococcus aureus (anti-MRSA) agents as initial antibiotic therapies, 28-day all-cause mortality, ICU-free days, ventilator-free days and adverse events. Patients are randomly assigned to receive Gram stain-guided treatment or guidelines-based treatment at a ratio of 1:1. In the Gram stain group, results of Gram staining of endotracheal aspirate are used to guide the selection of antibiotics. In the guidelines group, the combination of an anti-pseudomonal agent and an anti-MRSA agent is administered. A total sample size of 200 was estimated to provide a power of $80 \%$ with a one-sided alpha level of $2.5 \%$ and a non-inferiority margin of $20 \%$, considering $10 \%$ non-evaluable patients.

Discussion: The GRACE-VAP trial is expected to reveal whether Gram staining can reduce the use of broadspectrum antibiotics without impairing patient outcomes and thereby provide evidence for an antibiotic selection strategy in patients with VAP.

Trial registration: Clinicaltrials.gov, NCT03506113. Registered on 29 March 2018. University Hospital Medical Information Network, UMIN000031933. Registered on 26 March 2018.

Keywords: VAP, Gram staining, Antimicrobial therapy, Empirical therapy, Nosocomial infection, Mechanical ventilation, ICU, MDR, RCT

\footnotetext{
* Correspondence: k.yamakawa0911@gmail.com

${ }^{1}$ Division of Trauma and Surgical Critical Care, Osaka General Medical Center,

3-1-56 Bandai-Higashi, Sumiyoshi, Osaka 558-8558, Japan

Full list of author information is available at the end of the article
}

(c) The Author(s). 2018 Open Access This article is distributed under the terms of the Creative Commons Attribution 4.0 International License (http://creativecommons.org/licenses/by/4.0/), which permits unrestricted use, distribution, and reproduction in any medium, provided you give appropriate credit to the original author(s) and the source, provide a link to the Creative Commons license, and indicate if changes were made. The Creative Commons Public Domain Dedication waiver (http://creativecommons.org/publicdomain/zero/1.0/) applies to the data made available in this article, unless otherwise stated. 


\section{Background}

The increasing prevalence of infections caused by multidrug-resistant (MDR) organisms in intensive care units (ICUs) is recognised as a significant health threat all over the world [1, 2]. However, the development of new pharmaceutical agents is dwindling. Therefore, antibiotic options for the treatment of MDR organisms are becoming increasingly limited $[3,4]$. To alleviate this issue, optimising the use of antibiotic agents has been emphasised [5].

Antibiotics are extensively used in ICUs because of the increased risks of infection due to underlying diseases or conditions, impaired immunity, and exposure to multiple invasive devices. In fact, the Extended Prevalence of Infection in Intensive Care II study showed that $51 \%$ of patients were considered to be infected during their stay in ICUs [6]. Ventilator-associated pneumonia (VAP) is an important complication that generates a need for antibiotic administration in patients with mechanical ventilation because it is one of the most common hospital-acquired infections in ICUs [6-8]. Therefore, the overuse of broad-spectrum antibiotic agents in patients with VAP could be a major cause of the accelerated emergence of antimicrobial-resistant organisms [9]. The 2016 clinical practice guidelines [10] for VAP published by the Infectious Diseases Society of America (IDSA) and the American Thoracic Society (ATS) recommend that an empirical treatment should include coverage for methicillin-resistant Staphylococcus aureus (MRSA) and Pseudomonas aeruginosa. Although empirical broad-spectrum treatments help to ensure that infections are treated effectively, overuse of broad-spectrum antibiotic agents leads to an increase in antimicrobial-resistant organisms. Thus, there is an increasing need to establish methods to safely reduce the use of broad-spectrum antibiotic agents for VAP.

In this trial, we will adopt a traditional but credible method, the Gram stain, to estimate pathogens of VAP. Gram staining of respiratory samples is potentially useful to guide appropriate initial antibiotic therapy. Several studies have evaluated the correlation between the results of Gram staining and culture, but the results were conflicting [11-16]. Furthermore, the evidence for Gram stain-guided initial antibiotic treatment for VAP is not well established. Therefore, whether the results of Gram staining are accurate enough to safely restrict the use of broad-spectrum antibiotics is still controversial.

We hypothesise that antibiotic treatment based on Gram stain results can restrict the administration of broad-spectrum antibiotic agents for VAP without detrimental effects on patient outcomes. Thus, we planned the GRam stain-guided Antibiotics ChoicE for VAP (GRACE-VAP) trial to investigate whether Gram stain-guided antibiotic treatment is non-inferior to guidelines-based treatment with respect to the clinical response rate in patients with VAP.

\section{Methods/design}

\section{Design}

We will conduct a multicentre, open-label, randomised, non-inferiority trial with blinded endpoint assessment that will compare the Gram stain-guided treatment with standard antibiotic treatment for patients with VAP. The final trial report will follow the Consolidated Standards of Reporting Trials (CONSORT) statement and its extension to non-inferiority and equivalence trials [17]. This study was registered at clinicaltrials.gov under the registry number NCT03506113. The study protocol was written in accordance with the Standard Protocol Items: Recommendations for Interventional Trials (SPIRIT) guidelines (Additional file 1).

\section{Setting}

The study will be conducted in the ICUs of ten tertiary referral hospitals in Japan (Table 1).

\section{Patients}

All patients admitted to the ICU will be screened every day during their ICU stay by the study investigators. The study participants will be enrolled in this study if they meet all of the inclusion criteria and none of the exclusion criteria. Patients will be included if they (1) are aged 15 years or older; (2) have undergone mechanical ventilation for at least $48 \mathrm{~h}$; and (3) are diagnosed as having VAP, which is defined by a modified clinical pulmonary infection score of 5 or more [18].

Patients will be excluded if they (1) have an allergy to study medications, (2) are pregnant, (3) have already been discharged from the ICU, (4) are diagnosed as having heart failure or atelectasis, (5) have been administered antibiotics for more than $24 \mathrm{~h}$ when they meet the inclusion criteria, (6) are declined to provide full life support, or (7) are judged as inappropriate for inclusion at the discretion of the study physician.

Table 1 Participating institutions and investigators

\begin{tabular}{ll}
\hline Institution & Investigators \\
\hline Chukyo Hospital & Akinori Osuka, MD, PhD \\
Ebina General Hospital & Takeshi Yamagiwa, MD, PhD \\
Hitachi General Hospital & Kensuke Nakamura, MD, PhD \\
Kansai Medical University Hospital & Hiroki Takahashi, MD \\
Kansai Medical University Medical Center & Masahiro Kawada, MD \\
Nagasaki University Hospital & Shuhei Yamano, MD \\
Osaka General Medical Center & Jumpei Yoshimura, MD \\
Saga University Hospital & Hiroyuki Koami, MD, PhD \\
University of the Ryukyus Hospital & Takayuki Taira, MD \\
Wakayama Medical University Hospital & Kyohei Miyamoto, MD \\
\hline
\end{tabular}




\section{Ethics and informed consent}

The clinical trial will be carried out according to the principles of the Declaration of Helsinki and Ethical Guidelines for Medical and Health Research Involving Human Subjects published by the Ministry of Health, Labour and Welfare of Japan and the Japanese Ministry of Education, Culture, Sports, Science and Technology. The study protocol was approved by the ethics committees of each participating hospital. Written informed consent will be obtained from all patients or their representatives.

\section{Randomisation and allocation concealment}

Patients are randomly allocated to each treatment arm at a 1:1 ratio no earlier than 1 day before starting antibiotic therapy for VAP. Randomisation will be performed with the use of a stochastic minimisation procedure stratified by study centre, presence of chronic obstructive pulmonary disease, presence of traumatic brain injury, presence of post-cardiopulmonary arrest syndrome, and prior antibiotic therapy during the hospitalisation. We will use an electronic data capture system to conduct randomisation and data collection.

\section{Trial interventions}

Treatment of patients in this trial follows a pragmatic approach. All study medications are commercially available and approved in Japan. They are used as marketed in standard dosage regimens. According to their treatment group, patients will receive either antibiotics selected according to the results of Gram staining or standard therapy based on the IDSA/ATS guidelines [10] (Fig. 1).

In the Gram stain group, the results of Gram staining of endotracheal aspirate are used to guide the selection of antibiotics. The results of the Gram stains are categorised as Gram-positive cocci (GPC) chains, GPC clusters, Gram-positive bacilli (GPB), Gram-negative rods (GNR), or a combination of these. A non-pseudomonal $\beta$-lactam antibiotic is selected when the Gram stain of the endotracheal aspirate shows only GPC chains and/or GPB. An anti-MRSA agent is selected when the Gram stain results show GPC clusters without GNR. An anti-pseudomonal agent is selected when the Gram stain results show GNR without GPC clusters. The combination of an anti-pseudomonal agent and an anti-MRSA agent is selected when the Gram stain results show both GPC clusters and GNR. We escalate an initial treatment selection process to cover pathogens isolated from respiratory samples before the onset of VAP if the Gram stain results suggest their involvement.

In the standard group, patients are administered the combination of an anti-pseudomonal agent and an anti-MRSA agent according to IDSA/ATS guidelines, because $47.7 \%$ of $S$. aureus isolates are MRSA in Japanese ICUs (https://janis.mhlw.go.jp/report/open_report/2016/

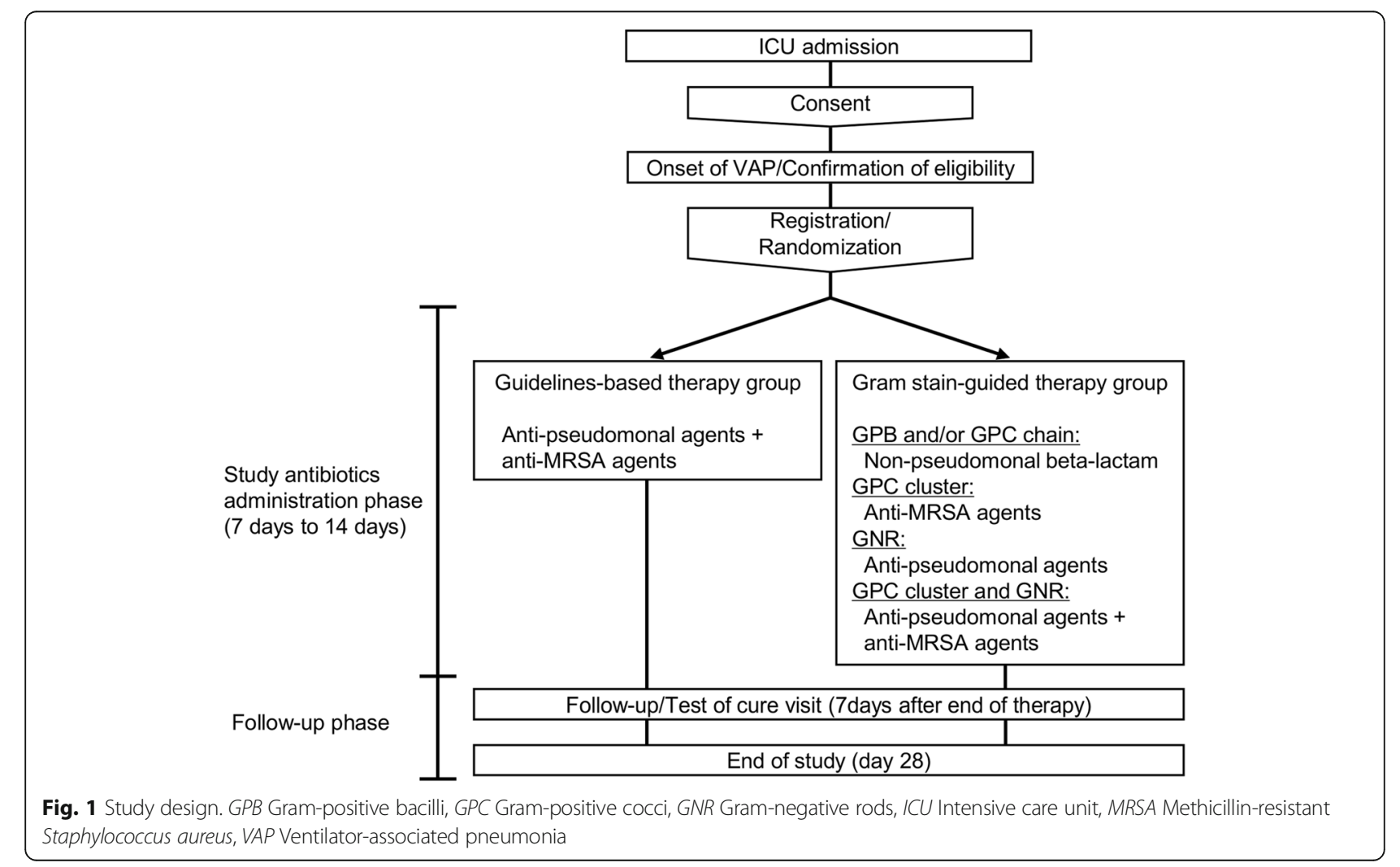


3/1/ken_Open_Report_201600.pdf). If drug-resistant pathogens are isolated from respiratory samples before the onset of VAP, we escalate an initial treatment selection process to cover them. In both groups, specific antibiotic agents are selected according to previously recorded antimicrobial resistance patterns in each ICU.

The study medication can be de-escalated or escalated to a definitive treatment level according to the results of the pathogens isolated from respiratory samples. Dose adjustments in individual patients are performed as judged appropriate by the site investigator. Study medications are continued for at least 7 days and discontinued on the basis of the site investigator's judgement.

\section{Assessment and follow-up}

Clinical assessments are performed at baseline and daily throughout the study treatment, at the end of therapy (EOT) and at the follow-up/test of cure (FU/TOC) visit (Fig. 2). Laboratory assessments such as renal function, liver function, platelet count and inflammatory markers including white blood cell count, C-reactive protein and procalcitonin will be performed on the randomisation day; days 2, 4, 6, 8 and 14; and at EOT. Endotracheal aspirates and blood samples for bacterial culture will be obtained on the day of randomisation. We will collect data on all pathogens isolated with at least 1+ semi-quantitative growth from endotracheal aspirates, all pathogens isolated from blood, and antibiotic sensitivity. We will also collect data on all antibiotic agents administered during the VAP treatment, the presence of escalation or de-escalation and the length of the antibiotic therapies. Patients are followed for 28 days to evaluate efficacy and safety variables. If a patient is discharged from hospital prior to 28 days after randomisation, the investigators will contact the patient (or the patient's representative as appropriate) by telephone to determine the patient's disposition and status.

\section{Outcome measures}

The primary outcome is the clinical response rate at FU/ TOC. Clinical response is defined as that fulfilling all four of the following components: completion of antibiotic therapy within 14 days, improvement or lack of progression of baseline radiographic findings at EOT, and resolution of signs and symptoms of pneumonia at FU/TOC. The signs and symptoms of pneumonia are defined as body temperature $\geq 38{ }^{\circ} \mathrm{C}$, increase in purulent sputum and deterioration of oxygenation. The site investigators will collect information relevant to the clinical response described above. On the basis of these individual data, blinded evaluators on the event adjudication committee will judge the achievement of primary outcome.

The secondary outcomes are the coverage rate of initial antibiotic therapies, the selected rates of anti-pseudomonal agents and anti-MRSA agents as initial antibiotic therapies, 28-day mortality, 28-day

\begin{tabular}{|c|c|c|c|c|c|c|}
\hline & ICU admission & Screening & Treatment & End of therapy & $\begin{array}{c}\text { Follow-up/ } \\
\text { test of cure visit }\end{array}$ & End of study \\
\hline Assessment Day & - & 1 & $2,4,6,8,14$ & - & $\begin{array}{l}7 \text { days after } \\
\text { end of therapy }\end{array}$ & 28 \\
\hline Informed consent & $\mathrm{x}$ & & & & & \\
\hline Check in/exclusion criteria & & $x$ & & & & \\
\hline Randomisation & & $\mathrm{x}$ & & & & \\
\hline Assessment of patient & & $\mathrm{x}$ & & & & \\
\hline Assessment of Gram stain & & $x$ & & & & \\
\hline Endotracheal aspirate culture & & $\mathrm{x}$ & & & & \\
\hline Blood culture & & $\mathrm{x}$ & & & & \\
\hline Laboratory data & & $\mathrm{x}$ & $\mathrm{x}$ & $\mathrm{x}$ & $\mathrm{x}$ & \\
\hline SOFA score & & $\mathrm{x}$ & $\mathrm{x}$ & $\mathrm{x}$ & & \\
\hline Chest X-ray & & $\mathrm{x}$ & & $\mathrm{x}$ & $\mathrm{x}$ & \\
\hline Clinical response (primary outcome) & & & & & $\mathrm{x}$ & \\
\hline Coverage of pathogenic bacteria & & & & $\mathrm{x}$ & & \\
\hline Assessment of antibiotic agents & & & & $\mathrm{x}$ & & \\
\hline Adverse events & & & $\mathrm{x}$ & $\mathrm{x}$ & $\mathrm{x}$ & \\
\hline 28-day ventilator-free days & & & & & & $\mathrm{x}$ \\
\hline 28-day ICU-free days & & & & & & $x$ \\
\hline 28-day mortality & & & & & & $\mathrm{x}$ \\
\hline
\end{tabular}

Fig. 2 Schedule of assessments. ICU intensive care unit, SOFA Sequential Organ Failure Assessment 
ICU-free days, 28-day ventilator-free days, and adverse events. Therapy will be considered appropriate when all pathogens isolated with at least $1+$ semi-quantitative growth from endotracheal aspirates are covered by the selected antibiotic agents. Safety outcomes are death during the study period and any adverse events reported at FU/ TOC, including renal impairment, thrombocytopenia, diarrhoea, Clostridium difficile infection, skin rash and seizure.

\section{Sample size}

The primary efficacy analysis will assess the non-inferiority of the clinical response of Gram stain-guided antibiotic therapy compared with standard therapy. The margin of non-inferiority is set at $20 \%$ on the basis of statistically acceptable tolerance and clinically acceptable margin. This margin was used in previous large clinical trials in the field of critical care [19-21]. Then, the non-inferiority of Gram stain-guided antibiotic therapy is concluded if the upper limit of the one-sided 97.5\% CI for the difference in clinical response (standard - Gram stain-guided) is less than $20 \%$. To achieve a power of $80 \%$ with an $\alpha$ level of $5 \%$, assuming as stated in the previous study that the clinical response rate is $67.8 \%$ with standard therapy and is the same with Gram stain-guided antibiotic therapy (unpublished data), with a non-inferiority margin of $-20 \%, 86$ patients are needed in each group. Assuming 10\% non-evaluable patients, we decided to enrol 100 patients per group.

\section{Statistical analysis}

Because this is a non-inferiority trial, we will compare the proportion of patients meeting the definition of clinical response using per-protocol analysis as a primary analysis. The per-protocol analysis population will consist of all randomised patients who are not lost to follow-up and have no major protocol deviations. We will also conduct an intention-to-treat analysis as a secondary analysis. We will attest the non-inferiority of the primary outcome on the basis of the normal theory test for binomial proportions. We will conduct the primary analysis without adjustment of potential confounders. We will construct multivariable logistic models or Cox proportional models including the stratified variables as the secondary analysis. We will conduct other post hoc exploratory analyses based on the recommendations of the steering committee. Because of the exploratory nature of these analyses for other than the primary endpoint, no correction for multiplicity is made.

Secondary outcomes will be analysed under a superiority or non-inferiority assumption, as appropriate. Pre-defined subgroup analyses for the primary and secondary endpoints include (1) patients who received prior antibiotic therapy during the hospitalisation versus those who did not, (2) length of ICU stay $\geq 5$ days versus < 5 days before randomisation, and (3) septic shock versus no septic shock.

All $P$ values are two-sided, and $P<0.05$ is considered significant other than for the non-inferiority test for clinical response, for which a one-sided $P<0.025$ is considered significant. All statistical analyses will be performed using JMP 13.1 and SAS 9.4 software (both from SAS Institute Inc., Cary, NC, USA).

\section{Trial oversight}

The trial will be managed by the Division of Trauma and Surgical Critical Care, Osaka General Medical Center, Osaka, Japan. The data centre is located at the Institute for Clinical Effectiveness, Kyoto, Japan, and data managers will centrally monitor the data through the study period. A steering committee was involved in protocol development and will oversee study progress (Table 2). A Data and Safety Monitoring Board (DSMB) made up of independent experts who are not involved in the conduct of the trial will oversee the safety of the trial and efficacy of the trial therapy and will monitor the integrity and validity of the data collected and the conduct of the clinical trial. A scheduled interim analysis will not be performed in this trial. The data centre will report to the steering committee monthly the numbers of registration and mortality as well as occurrence of serious adverse events. When the absolute difference in mortality becomes greater than five patients or any concerns develop, the steering committee will consult the DSMB on the need for an interim analysis. The DSMB will independently perform the interim analysis and make a recommendation to the steering committee whether to continue the trial.

\section{Discussion}

The GRACE-VAP trial will assess whether initial Gram stain-based restriction of antibiotic therapy is non-inferior to guidelines-based initial broad-spectrum antibiotic therapy in patients with VAP in terms of the clinical response rate. If non-inferiority is achieved, the Gram stain-guided antibiotic choice will be considered to have several advantages in clinical settings. First, Gram staining can be a point-of-care test for selecting antibiotic agents because it should be easy to access at the bedside clinically. Second, it is an inexpensive examination and easy to install in ICUs all over the world, including developing countries. Third, it is easy to learn how to evaluate the results of Gram staining by the method indicated in this trial because we apply merely a simple classification of bacteria.

To the best of our knowledge, this trial will be the first study to answer the clinical question of whether Gram 
Table 2 Study oversight

\begin{tabular}{|c|c|c|}
\hline Role in study & Name & Institution \\
\hline Principal investigator & \multirow[t]{2}{*}{ Jumpei Yoshimura, MD } & \multirow{2}{*}{$\begin{array}{l}\text { Division of Trauma and Surgical Critical Care, Osaka General } \\
\text { Medical Center }\end{array}$} \\
\hline Steering Committee & & \\
\hline Steering Committee & Kazuma Yamakawa, MD, PhD & $\begin{array}{l}\text { Division of Trauma and Surgical Critical Care, Osaka General } \\
\text { Medical Center }\end{array}$ \\
\hline Steering Committee & Takeshi Morimoto, MD, PhD, MPH & $\begin{array}{l}\text { Department of Clinical Epidemiology, Hyogo College of } \\
\text { Medicine }\end{array}$ \\
\hline Steering Committee & Yoshinori Ohta, MD & $\begin{array}{l}\text { Division of General Medicine, Department of Internal Medicine, } \\
\text { Hyogo College of Medicine }\end{array}$ \\
\hline Event Adjudication Committee & Kazuma Yamakawa, MD, PhD & $\begin{array}{l}\text { Division of Trauma and Surgical Critical Care, Osaka General } \\
\text { Medical Center }\end{array}$ \\
\hline Event Adjudication Committee & Hiroki Takahashi, MD & Kansai Medical University Hospital \\
\hline Event Adjudication Committee & Kyohei Miyamoto, MD & Wakayama Medical University Hospital \\
\hline Data and Safety Monitoring Board & Yuichiro Oba, MD, DTM\&H & $\begin{array}{l}\text { Department of General Internal Medicine and Infectious Diseases, } \\
\text { Osaka General Medical Center }\end{array}$ \\
\hline Data and Safety Monitoring Board & Atsushi Hiraide, MD, PhD & Critical Care Medical Center, Faculty of Medicine, Kindai University \\
\hline Data and Safety Monitoring Board & Hitoshi Yamamura, MD, PhD & $\begin{array}{l}\text { Department of Critical Care Medicine, Graduate School of Medicine, } \\
\text { Hirosaki University }\end{array}$ \\
\hline Study statistician & Takeshi Morimoto, MD, PhD, MPH & Department of Clinical Epidemiology, Hyogo College of Medicine \\
\hline Study secretariat & - & $\begin{array}{l}\text { Division of Trauma and Surgical Critical Care, Osaka General Medical } \\
\text { Center }\end{array}$ \\
\hline Project management & - & $\begin{array}{l}\text { Division of Trauma and Surgical Critical Care, Osaka General Medical } \\
\text { Center }\end{array}$ \\
\hline Data management & - & Institute for Clinical Effectiveness \\
\hline
\end{tabular}

staining can reduce the use of broad-spectrum antibiotics without impairing patient outcomes. We hope that this trial will have a great impact on the establishment of a novel strategy to optimise the use of antibiotic agents safely and restrict the overuse of broad-spectrum antibiotic agents.

\section{Trial status}

The first patient was recruited on 1 April 2018. The last patient is expected to be recruited in March 2021. Osaka General Medical Center provides central trial management and coordination.

\section{Additional file}

Additional file 1: SPIRIT checklist. (DOC $121 \mathrm{~kb}$ )

\footnotetext{
Abbreviations

ATS: American Thoracic Society; DSMB: Data and Safety Monitoring Committee; EOT: End of therapy; FU/TOC: Follow-up/test of cure visit; GNR: Gram-negative rods; GPB: Gram-positive bacilli; GPC: Gram-positive cocci; GRACE-VAP: GRam stain-guided Antibiotics ChoicE for VentilatorAssociated Pneumonia trial; ICU: Intensive care unit; IDSA: Infectious Diseases Society of America; MDR: Multidrug-resistant; MRSA: Methicillin-resistant Staphylococcus aureus; VAP: Ventilator-associated pneumonia
}

\section{Acknowledgements}

We would like to thank all investigators for their effort in conducting the GRACE-VAP trial.

\section{Funding}

Funding is provided by the Division of Trauma and Surgical Critical Care, Osaka General Medical Center. However, the funding source had no role in 1) study design; 2) the collection, analysis, and interpretation of data; 3) the writing of the report; and 4) the decision to submit the manuscript for publication.

\section{Authors' contributions}

JY conceived of and designed this study and drafted the manuscript. KY conceived of and designed this study and was responsible for drafting, editing and submission of the manuscript. TK critically contributed to the concept and design of the study. YO contributed to the design of the study and reviewed the manuscript. TM had a major influence on the design of this study, such as statistical and methodological expertise, and helped in reviewing and revising the manuscript for intellectual content. All authors read and approved the final manuscript.

\section{Ethics approval and consent to participate}

The protocol and consent forms were approved by the institutional review boards at Osaka General Medical Center and each of the participating institutions. All substantial protocol modifications will be notified as protocol amendments to them. All patients or their legally authorised representatives will provide written informed consent before randomisation by the study physicians.

\section{Consent for publication}

Not applicable.

\section{Competing interests}

The authors declare that they have no competing interests. 


\section{Publisher's Note}

Springer Nature remains neutral with regard to jurisdictional claims in published maps and institutional affiliations.

\section{Author details}

'Division of Trauma and Surgical Critical Care, Osaka General Medical Center, 3-1-56 Bandai-Higashi, Sumiyoshi, Osaka 558-8558, Japan. 'Division of General Medicine, Department of Internal Medicine, Hyogo College of Medicine, 1-1 Mukogawa, Nishinomiya, Hyogo 663-8501, Japan. ${ }^{3}$ Department of Clinical Epidemiology, Hyogo College of Medicine, 1-1 Mukogawa, Nishinomiya, Hyogo 663-8501, Japan.

Received: 10 July 2018 Accepted: 8 October 2018 Published online: 08 November 2018

\section{References}

1. Llor C, Bjerrum L. Antimicrobial resistance: risk associated with antibiotic overuse and initiatives to reduce the problem. Ther Adv Drug Saf. 2014;5: 229-41

2. World Health Organisation (WHO). Antimicrobial resistance: global report on surveillance. 2014. http://apps.who.int/iris/bitstream/10665/112642/1/ 9789241564748 eng.pdf. Accessed 24 Oct 2018.

3. Wenzel RP. The antibiotic pipeline-challenges, costs, and values. N Engl J Med. 2004:351:523-6.

4. Cassell GH, Mekalanos J. Development of antimicrobial agents in the era of new and reemerging infectious diseases and increasing antibiotic resistance. JAMA. 2001;285:601-5.

5. World Health Organisation (WHO). Global action plan on antimicrobial resistance. 2015. http://apps.who.int/iris/bitstream/10665/193736/1/ 9789241509763_eng.pdf. Accessed 24 Oct 2018.

6. Vincent JL, Rello J, Marshall J, Silva E, Anzueto A, Martin CD, et al. International study of the prevalence and outcomes of infection in intensive care units. JAMA. 2009:302:2323-9.

7. Ponce de Leon-Rosales SP, Molinar-Ramos F, Dominguez-Cherit G, RangelFrausto MS, Vazquez-Ramos VG. Prevalence of infections in intensive care units in Mexico: a multicentre study. Crit Care Med. 2000;28:1316-21.

8. Erbay H, Yaicin AN, Serin S, Turgut H, Tomatir E, Cetin B, et al. Nosocomial infections in intensive care unit a Turkish university hospital: 2-year survey. Intensive Care Med. 2003:29:1482-8.

9. Laxminarayan R, Duse A, Wattal C, Zaidi AK, Wertheim HF, Sumpradit N, et al. Antibiotic resistance - the need for global solutions. Lancet Infect Dis. 2013;12:1057-98.

10. Kalil AC, Metersky ML, Klompas M, Muscedere J, Sweeney DA, Palmer LB, et al. Management of adults with hospital-acquired and ventilatorassociated pneumonia: 2016 clinical practice guidelines by the Infectious Diseases Society of America and the American Thoracic Society. Clin Infect Dis. 2016;63:e61-e111.

11. Yoshimura J, Kinoshita T, Yamakawa K, Matsushima A, Nakamoto N, Hamasaki T, et al. Impact of Gram stain results on initial treatment selection in patients with ventilator-associated pneumonia: a retrospective analysis of two treatment algorithms. Crit Care. 2017;21:156

12. Seligman R, Seligman BG, Konkewicz L, Dos Santos RP. Accuracy of tracheal aspirate gram stain in predicting Staphylococcus aureus infection in ventilator-associated pneumonia. BMC Anesthesiol. 2015:15:19.

13. Gottesman T, Yossepowitch O, Lerner E, Schwartz-Harari O, Soroksky A, Yekutieli D, et al. The accuracy of Gram stain of respiratory specimens in excluding Staphylococcus aureus in ventilator-associated pneumonia. J Crit Care. 2014;29:739-42.

14. O'Horo JC, Thompson D, Safdar N. Is the gram stain useful in the microbiologic diagnosis of VAP? A meta-analysis. Clin Infect Dis. 2012;55: 551-61.

15. Tetenta S, Metersky ML. Tracheal aspirate Gram stain has limited sensitivity and specificity for detecting Staphylococcus aureus. Respirology. 2011;16:86-9.

16. Blot F, Raynard B, Chachaty E, Tancrede C, Antoun S, Nitenberg G. Value of gram stain examination of lower respiratory tract secretions for early diagnosis of nosocomial pneumonia. Am J Respir Crit Care Med. 2000;162: 1731-7.

17. Piaggio G, Elbourne DR, Pocock SJ, Evans SJ, Altman DG; CONSORT Group. Reporting of noninferiority and equivalence randomized trial: extension of the CONSORT 2010 statement. JAMA. 2012;308:2594-604.
18. Luna CM, Blanzaco D, Niederman MS, Matarucco W, Baredes NC, Desmery P, et al. Resolution of ventilator-associated pneumonia: prospective evaluation of the clinical pulmonary infection score as an early clinical predictor of outcome. Crit Care Med. 2003:31:676-82.

19. Fowler VG Jr, Boucher HW, Corey GR, Abrutyn E, Karchmer AW, Rupp ME, et al. S. aureus Endocarditis and Bacteremia Study Group. Daptomycin versus standard therapy for bacteremia and endocarditis caused by Staphylococcus aureus. N Engl J Med. 2006;355:653-65.

20. Chastre J, Wunderink R, Prokocimer P, Lee M, Kaniga K, Friedland I. Efficacy and safety of intravenous infusion of doripenem versus imipenem in ventilator-associated pneumonia: a multicenter, randomized study. Crit Care Med. 2008;36:1089-96.

21. Rubinstein E, Lalani T, Corey GR, Kanafani ZA, Nannini EC, Rocha MG, ATTAIN Study Group, et al. Telavancin versus vancomycin for hospitalacquired pneumonia due to gram-positive pathogens. Clin Infect Dis. 2011; $52: 31-40$

Ready to submit your research? Choose BMC and benefit from:

- fast, convenient online submission

- thorough peer review by experienced researchers in your field

- rapid publication on acceptance

- support for research data, including large and complex data types

- gold Open Access which fosters wider collaboration and increased citations

- maximum visibility for your research: over $100 \mathrm{M}$ website views per year

At BMC, research is always in progress.

Learn more biomedcentral.com/submissions 